WHITE PAPER FOR THE

PLANETARY SCIENCE DECADAL SURVEY OF 2023-2032

\title{
Venus Corona and Tessera Explorer (VeCaTEx)
}

\author{
Primary author: James A. Cutts \\ Jet Propulsion Laboratory, California Institute of Technology \\ Phone: (818)354-4120 \\ E-Mail: James.A.Cutts@jpl.nasa.gov
}

\section{Co-authors}

Kevin Baines ${ }^{1}$

Patricia Beauchamp ${ }^{1}$

Chad Bower ${ }^{2}$

Anthony Davis ${ }^{1}$

Len Dorsky ${ }^{1}$

Darby Dyar ${ }^{3,4}$

Lorraine Fesq ${ }^{1}$

Anthony Freeman ${ }^{1}$

Richard Ghail ${ }^{5}$

Co Signees

Ratnakumar Bugga
Martha Gilmore 6

Robert Grimm ${ }^{7}$

Anna Gülcher ${ }^{8}$

James Head ${ }^{9}$

Joern Helbert ${ }^{10}$

Jennifer Jackson ${ }^{11}$

Maxim De Jong, ${ }^{12}$

Jeffery L. Hall ${ }^{1}$

Jacob Izraelevitz ${ }^{1}$

Jonathan Grandidier
Siddharth Krishnamoorthy ${ }^{1}$ Larry Matthies ${ }^{1}$ Laurent Montesi ${ }^{13}$ Michael T. Pauken ${ }^{1}$

David Senske ${ }^{1}$

Christophe Sotin ${ }^{1}$

Brian Sutin ${ }^{1}$

Colin Wilson ${ }^{14}$

\section{Institutions}

${ }^{1}$ Jet Propulsion Laboratory, California

${ }^{9}$ Brown University

Institute of Technology

${ }^{10}$ German Aerospace Center (DLR)

2Paragon Space Development Corporation

${ }^{11}$ Division of Geological and Planetary

${ }^{3}$ Planetary Science Institute

Science, California Institute of Technology

${ }^{4}$ Mount Holyoke University

${ }^{12}$ Thin Red Line Aerospace

${ }^{5}$ Royal Holloway, University of London

${ }^{13}$ University of Maryland

${ }^{6}$ Wesleyan University

${ }^{14}$ Dept. of Physics, University of Oxford

${ }^{7}$ South West Research Institute

${ }^{15}$ Lunar and Planetary Institute

\section{${ }^{8}$ ETH Zurich}

Pre-Decisional Information - For Planning and Discussion Purposes Only. Part of the research was carried out at the Jet Propulsion Laboratory, California Institute of Technology, under a contract with the National Aeronautics and Space Administration (80NM0018D0004).Copyright 2020. All rights reserved.

Government sponsorship is acknowledged 
INTRODUCTION: The goal of the Venus Corona and Tessera Explorer (VeCaTEx) is to study the two feature categories that are believed to record the earliest geological events on the planet and the most recent volcanic and tectonic activity. VeCaTEx would acquire near infrared images of the surface of Venus with a special focus on coronae and tesserae with the object of learning more about the most ancient rocks on Venus exposed in the tessera and the most recent volcanic events taking place within the coronae. VeCaTEX accomplishes this by acquiring these images from an aerobot operating beneath the base of the Venus clouds thereby avoiding the degradation in spatial resolution of infrared signatures viewed from orbit. The spectral information would characterize the iron contents and infer rock types of the surface of Venus at high spatial resolution. Stereoscopic images at multiple wavelengths would enable stratigraphic relationships to be identified thereby advancing knowledge of the geological history of the planet. In addition to surface images, VeCaTEX could also image gas plumes resulting from outgassing in regions of recent volcanic activity. This paper describes the scientific motivation for the VeCaTEx mission concept and advanced aerobot technologies that the Venus Exploration Assessment Group (VEXAG) [1] determined could be ready for a launch to Venus early in the decade 2033-2042.

SCIENTIFIC MOTIVATION: The concept of remotely mapping the composition of the surface of Venus by observing thermal emission on the nightside of Venus, in a set of visible and near infrared spectral windows, emerged from Venus flyby observations by the Galileo and Cassini spacecraft [2], [3], [4], [5]. When improved data from ESA's Venus Express mission became available, areas of enhanced near infrared emissivity were identified and correlated with volcanic features identified in radar images (Figure 1 a). Because increased emissivity associated with the eruption of fresh volcanic rocks is not expected to persist in the corrosive Venus surface environment, these deposits are likely less than a million years old [6].

Other lines of evidence have recently been used to make the case that very young volcanic activity is widespread on Venus [7]. Several coronae, characterized by concentric rings of fractures (Figure $1 \mathrm{~b}$ ), appear to be among the youngest features on the planet. These coronae are likely to have formed by the interaction of mantle plumes with the lithosphere. Recent models of this interaction [7] and comparisons with topography indicate that currently active, hot mantle plumes are shaping numerous coronae on Venus. This implies that these coronae have been active both volcanically and tectonically in the last million years.

Spectral imaging is not only a vital tool for identifying and investigating recent volcanic activity on Venus but is also the key to characterizing lithological differences between the plains and the tesserae, regions of highly deformed terrain believed to date from very early in the history of the planet. Imaging at $1.02 \mu \mathrm{m}$ of Alpha Regio, an extensive tessera region near $20^{\circ} \mathrm{S}$ and $15^{\circ} \mathrm{E}$, has revealed a composition distinct from that of the surrounding plains (Figure 2) and indicative of high silica composition [8].

In summary, emissivity data are critical for identifying recent volcanism particularly in the youthful coronae as well as the very oldest features - the tesserae. As a result of the vital importance of this type of data, a Venus Emissivity Mapper (VEM), is included in the VERITAS mission concept proposed to NASA's Discovery program [9], the EnVision mission concept, which 
is a candidate for ESA's M5 opportunity [10], and the Venus Flagship Mission concept [11]. In the first two cases case, VEM would provide near global coverage of these IR signatures.

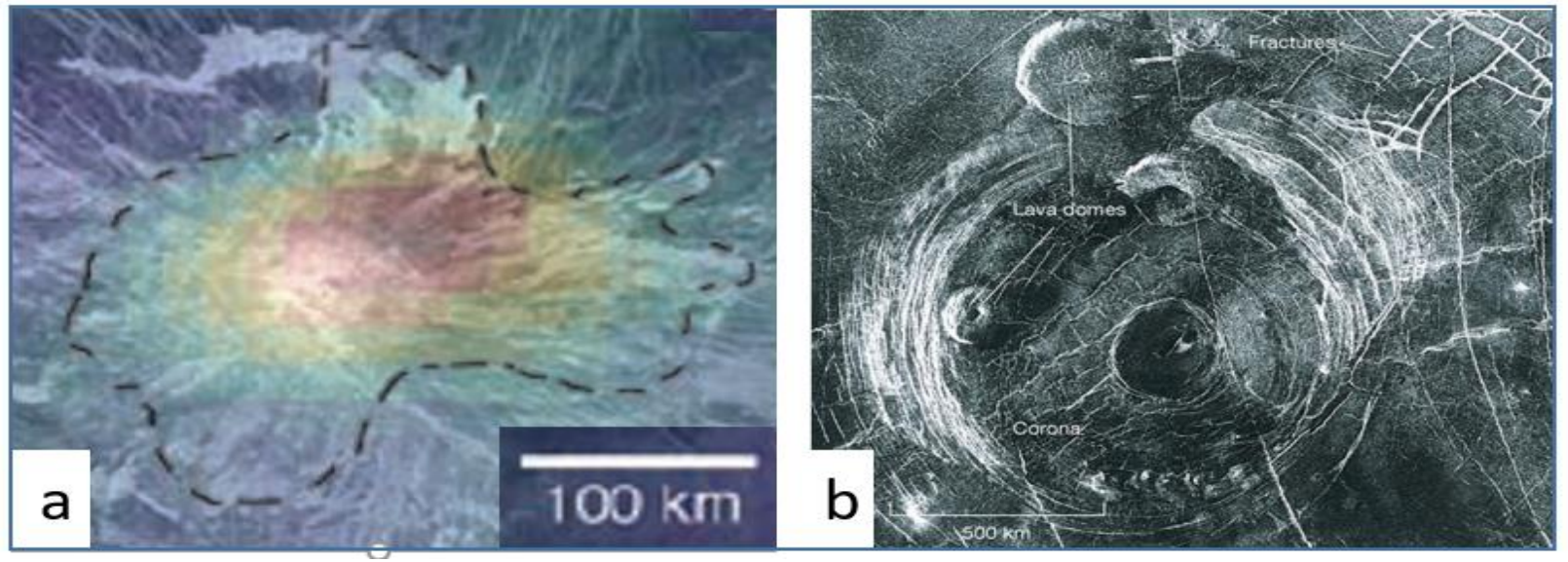

Figure 1 (a) The positive emissivity anomaly (orange color) measured at $1.02 \mu$ m covers the summit of Idunn Mons shown in the underlying radar image located near 46S, 215E reproduced from Smrekar [6]. The limited resolution of the emissivity map (100km) cannot discriminate between and anomaly localized in narrow flows or in a broadly dispersed deposit. (b) Corona Ainu feature with circumferential fractures and lava domes. Corona features may result from interaction of mantle plumes with the lithosphere

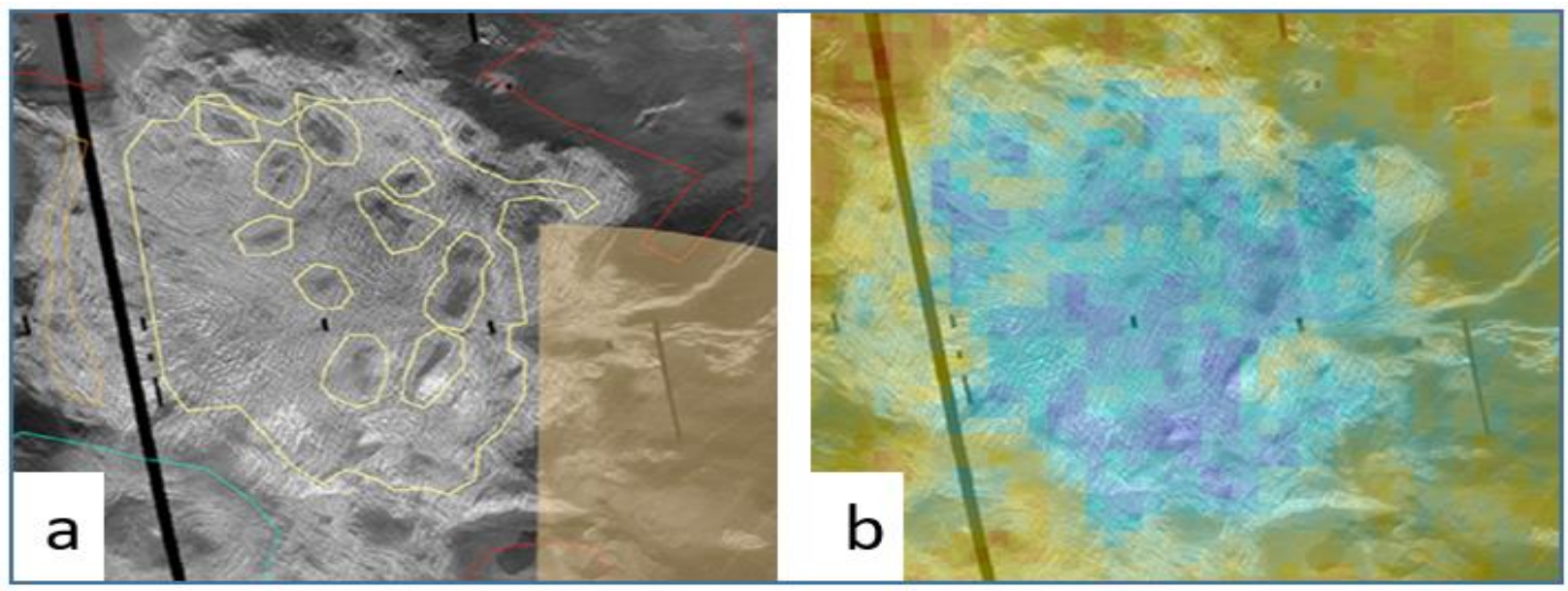

Figure 2 Alpha Regio, a tessera region of Venus, which is about $1500 \mathrm{~km}$ across and sits 1 to $2 \mathrm{~km}$ higher than the surrounding terrain viewed in a radar image (a) that is overlain with an infrared emissivity image (b). (reproduced from [8]). Alpha Regio is large enough that their overall shape is expressed in the emissivity image but the resolution is insufficient to provide much information about the distribution of interior geologic units.

While VEM's global map is clearly the next step in the exploration of Venus, it is important to recognize that the spatial resolution of the orbital infrared emissivity maps will be limited to the $100 \mathrm{~km}$ achieved by Galileo/NIMS, Cassini/VIMS, and Venus Express/VIRTIS. This limit is determined by optical scattering in the thick layer of continuous cloud extending from an altitude of about 48 to $70 \mathrm{~km}$ that lies between the orbital spacecraft and the surface of Venus. This limit is inherent and advances in orbital instrumentation cannot improve upon it. VeCaTEx looks beyond the limitations of orbital platforms to provide a method of acquiring emissivity maps with spatial resolutionin the near infra red (NIR) comparable to that of existing radar data or better. 
VeCaTEx incorporates a Type 3 aerobot described in a companion white paper [12] capable of penetrating below the cloud base from where it images the Venus surface.

SURFACE IMAGING FROM BENEATH THE CLOUDS: Beneath the clouds, particulate and aerosol scattering is largely absent but a deep Raleigh scattering atmosphere still impedes imaging at visual and near infrared wavelengths and gaseous absorption features limit surface imaging to narrow spectral windows. The left panel of Figure 3 depicts the Rayleigh optical depth in three of the spectral windows that provide access to surface emission. Because of the inverse fourth power dependence of the Rayleigh scattering cross section on wavelength, the optical depth varies dramatically from 3.7 at $0.82 \mu \mathrm{m}$ to 0.7 at $1.18 \mu \mathrm{m}$. The gradient of variation of the optical depth with altitude directly beneath the clouds is quite shallow and even at $20 \mathrm{~km}$ the optical depth is roughly $70 \%$ of the value beneath the clouds.
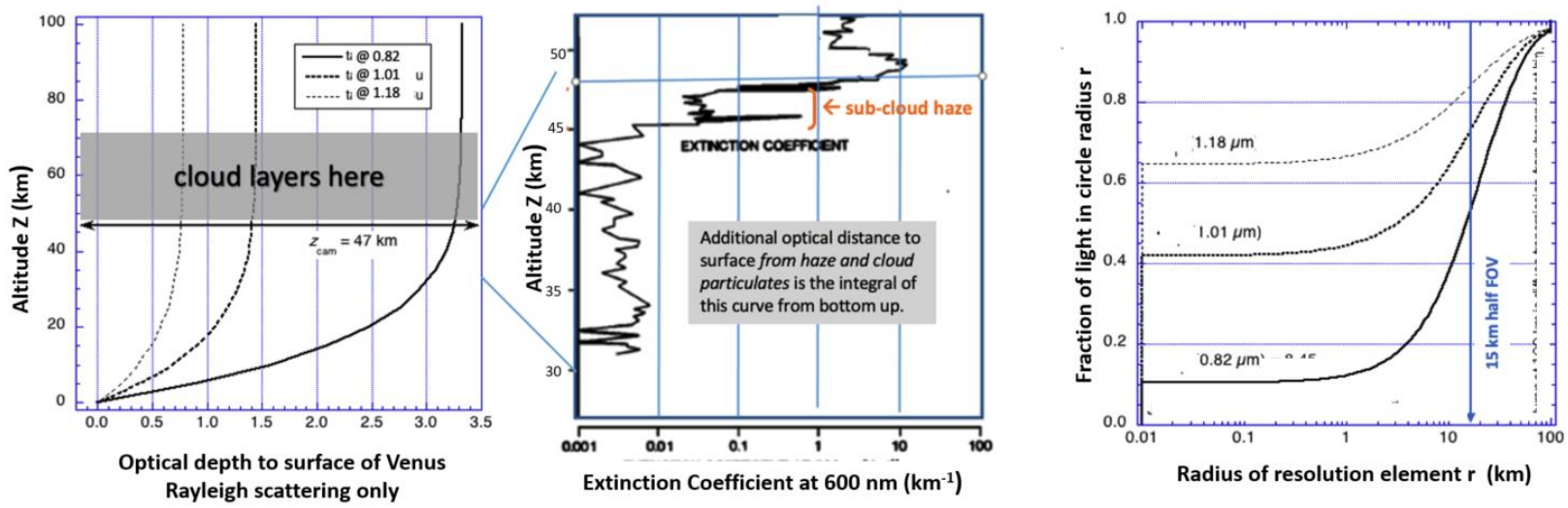

Figure 3 Impact of atmospheric scattering on Venus surface imaging from below the clouds (a) Optical depth as a function of altitude at three wavelengths 0.8, 1.0 and 1.2 $\mu \mathrm{m}$ with Rayleigh scattering only. (b) Extinction coefficient from haze particles below the clouds from [9] (c) Comparison of sub-cloud imaging at three wavelengths with imaging from orbit-Rayleigh scattering only.

In addition to Rayleigh scattering, there is also scattering from small haze particles according to measurements made by the large-probe cloud particle size spectrometer (LCPS) on NASA's Venus Pioneer mission [13]. Below $45 \mathrm{~km}$, small particles account for an extinction coefficient at $0.6 \mu \mathrm{m}$ of less than 0.1 - and potentially another 0.1 between $45 \mathrm{~km}$ and $48 \mathrm{~km}$ (Figure $3 \mathrm{~b}$ ).

The impact of Rayleigh scattering alone on the achievable spatial resolution from the sub-cloud region at the same three wavelength appears in Figure 3 (c), assuming a camera aperture of at least $1 \mathrm{~cm}$. At $1.18 \mu \mathrm{m}$, almost $63 \%$ of the emitted radiation is not scattered by the atmosphere and can be focused on a pixel with a projected size of $10 \mathrm{~m}$ on the Venus surface. The scattered radiation produces a halo around this image extending to tens of kilometers. At $1.01 \mu \mathrm{m}, 41 \%$ of the radiation is within the $10 \mathrm{~m}$ pixel. However, at $0.82 \mu \mathrm{m}, 90 \%$ of the radiation is scattered into a halo but with $50 \%$ of the light still contained within a radius of $15 \mathrm{~km}$. While images at $0.82 \mu \mathrm{m}$ would be greatly inferior to those at $1.18 \mu \mathrm{m}$, they will still be superior to orbital images that are limited to $100 \mathrm{~km}$ resolution by the cloud scattering (Figure 3 (c). Haze particles below $45 \mathrm{~km}$ will contribute negligible further degradation but the contribution between 45 and $47 \mathrm{~km}$ and near surface hazes [14] is being evaluated [15]. Rayleigh scattering of light reflected back from the lower part of the main cloud will also degrade contrast and is also being assessed. Daytime images at $1.18 \mu \mathrm{m}$ may also be feasible of low contrast but with higher signal levels. 
AEROBOT CAPABILITIES: A companion white paper [12], describes three types of Venus aerobot: Type 1 operates at a fixed altitude typically near $55 \mathrm{~km}$; Type 2 has the capability of changing altitude within the cloud but without exposure to temperatures outside the range for typical spacecraft hardware; and Type 3 is capable of reaching the cloud base. VeCaTEx requires the capabilities of the Type 3 aerobot that enables unique science resulting from penetrating below the cloud base. One implementation of a Type 3 aerobot is currently being studied by Paragon Corporation [16] in the form of a mechanical compression aerobot (Figure 4 a).

The VeCaTEx aerobot will descend for short periods from cooler regions of the atmosphere above $55 \mathrm{~km}$ to the high temperature region below the cloud base in order acquire data and then return to altitude to relay data to Earth and recharge the thermal control system (Figure $4 \mathrm{~b}$ ). The mechanical compression aerobot would use materials capable of surviving and operating in the sub cloud environment. Its slender profile permits rapid descents at up to $10 \mathrm{~m} / \mathrm{s}$ from the cool mid-cloud region to the cloud base. Energy estimates from [17] for similar altitude ranges indicate the need for substantial energy storage capability on the aerobot.

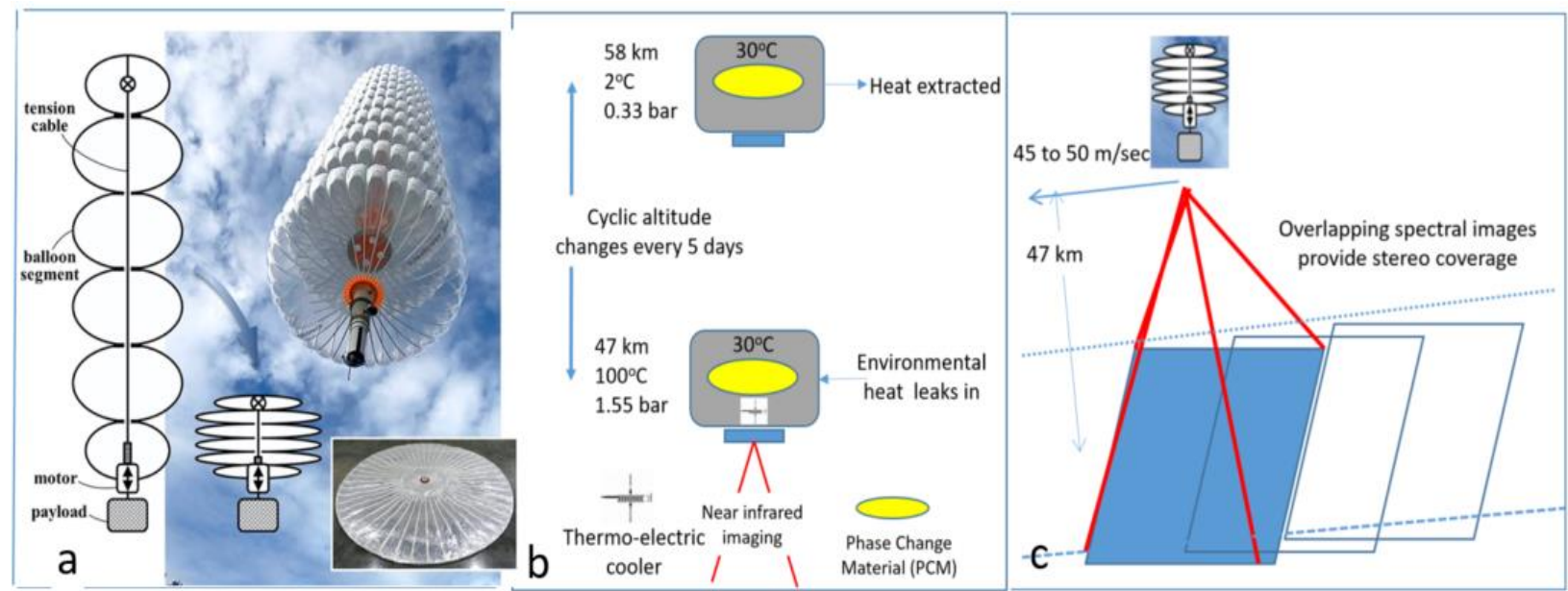

Figure 4 Key elements of VeCaTEx concept a) The Mechanical Compression aerobot. Balloon volume and hence buoyancy is changed by applying tension to a tether connecting the segments. b) Thermal control approach using phase change material and thermal electric cooler for the NIR sensor c) Motion of aerobot is used to generate a swathe of stereo NIR images.

INSTRUMENTATION: The primary instrument is an image-stabilized cameras equipped with 3000 $X 3000$ size array sensitive with filters for the key atmospheric windows at $1.18 \mu \mathrm{m}, 1.01 \mu \mathrm{m}$ and $0.82 \mu \mathrm{m}$. The camera field of view is adequate to provide a swath width of $30 \mathrm{~km}$ on the Venus surface from the observational altitude of $47 \mathrm{~km}$ (Figure $4 \mathrm{c}$ ). The pixel size would be about $10 \mathrm{~m}$. Images would be acquired approximately every 200 seconds as the aerobot advances $15 \mathrm{~km}$ along the ground track. Overlap between successive images would provide false color stereoscopic imaging to enhance the utility of the data for geological interpretation. The surface radiance viewed from below the clouds is 10 to 15 times higher than from orbit. Accordingly, at $1.18 \mu \mathrm{m}$ with a 5-nm filter an exposure time of only $50 \mathrm{~ms}$ for a $\mathrm{S} / \mathrm{N}$ of 100:1. However, at 0.82 $\mu \mathrm{m}$ the comparable exposure is 2.5 second. The average motion of the aerobot over the surface would be 45 to $50 \mathrm{~m} / \mathrm{s}$. For the shorter wavelengths, Time Delayed Integration (TDI) as well as Image Motion Compensation (IMC) controlled by an Inertial Measurement Unit (IMU) would provide images of adequate signal to noise when the aerobot platform is subject to turbulence. 
In addition to surface images, plumes of water vapor and other gases could be detected close to the surface (narrow filter at $1.17 \mu \mathrm{m}$ ) [10] and correlated with surface features.

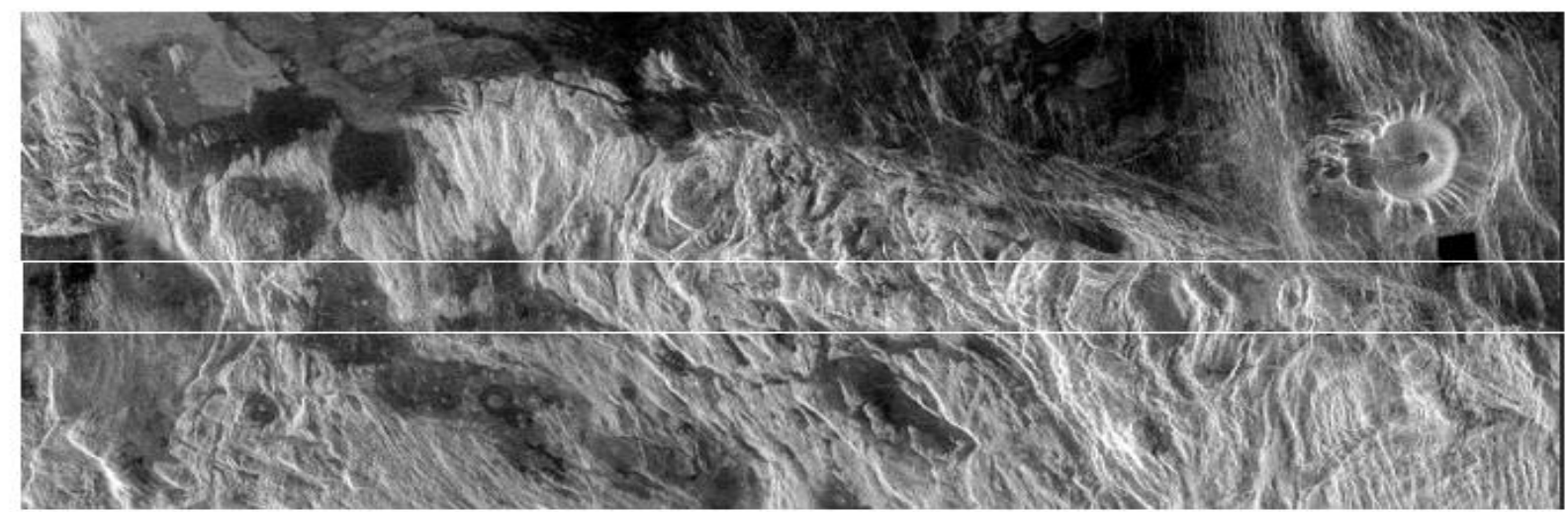

Figure 5 Radar image of the northern part of a major tessera region Alpha Regio located near 20 S and $15 E$ on Venus also seen in Figure 2. Tick volcano is the 30-km diameter crate at the upper right. The two parallel white lines illustrate the width of the infrared image swath acquired as the aerobot passes over Alpha Regio propelled by the super rotating flow. The resolution of the infrared images will be a factor of 10 better than the background radar images seen in this figure but comparable to the radar images expected by the time VeCaTEx is deployed to Venus.

CONCEPT OF OPERATIONS: The following notional sequence of operations has been defined for each circumnavigation of the planet by the aerobot:

1. Recharge the aerobot battery with solar energy during the dayside pass at its staging altitude

2. Adjust altitude to refine aerobot trajectory for next surface target

3. After the aerobot crosses over to the night side complete cooling of the payload elements including phase change materials

4. Approximately 15 minutes before arrival over the target initiate descent

5. Begin the imaging traverse. Imaging operations should be feasible for up to 4 hours for each circumnavigation of Venus allowing a strip of $\sim 1000 \mathrm{~km}$ to be covered

6. Immediately after image acquisition is complete, aerobot ascends to its staging altitude

7. Begin the process of cooling the systems

8. Begin relay of data to the orbiter

9. Execute altitude changes to optimize aerobot trajectory for next imaging traverse

Assuming stereo images in four filters ( 3 surface, one plume) with lossless compression, 2.4 Gbits of data would be acquired in each traverse. Transmission to a Venus orbiter at a data rate of 20 $\mathrm{kb} / \mathrm{s}$ would require an average of 33 hours of the approximately 65 available during the daylight period for each aerobot circumnavigation of Venus. Because the orbit alignments may not always be favorable for data relay, data would be stored on the aerobot for relay when those alignments are optimal. The above estimates reflect the poorer spatial resolution of images at wavelengths shortward of $1.02 \mu \mathrm{m}$ that will reduce data return requirements for those images. For a 100-day mission approximately 20 traverses of different targets would be acquired.

SCIENTIFIC IMPACT: A VeCaTex mission acquiring 20 stereo profiles across Venus each covering $1000 \times 30 \mathrm{~km}$ in several NIR bands and with resolution of $10 \mathrm{~m} /$ pixel will address directly six of the key investigations [1] identified by the Venus Exploration Analysis Group complementing those accomplished by the Mid Cloud Type 2 aerobot (see companion white paper [18]) 


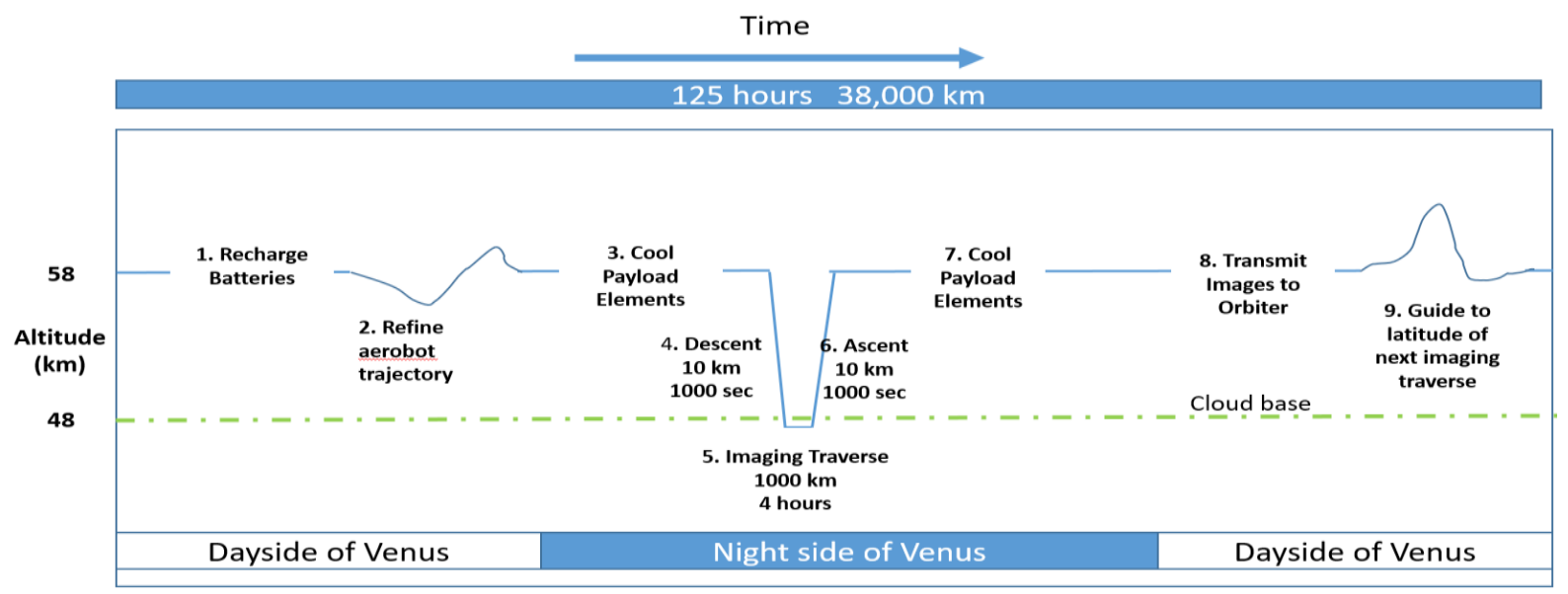

Figure 6 Trajectory of the VEXATEx aerobot during a single navigation around the planet. The trajectory is shown with time increasing to the right although the motion of the aerobot in the superrotating retrograde flow is towards the west on Venus.

Table 1 VEXAG Goals Objectives and Investigations (GOI) addressed by the VEXATEX Mission Concept

\begin{tabular}{|c|c|}
\hline Code & Description \\
\hline I.A. Hо & $\begin{array}{l}\text { Hydrous Origins: Determine whether Venus shows evide nce for abundant silicic igneous rocks and/or ancient } \\
\text { sedimentary rocks }\end{array}$ \\
\hline I.A.RE & Recycling: Search for st ructural, ge omorphic and che mical evide nce for crustal recycling on Venus \\
\hline II.B.OG & Outgassing: Dete rmine the products of volcanic outgassing on Venus and their effect on atmosphe ric composition \\
\hline III.A.GH & $\begin{array}{l}\text { Geologic History: Develop a geologic history for Venus by characte rizing the stratigraphy, modification state and } \\
\text { relative ages of surface units. }\end{array}$ \\
\hline III.A.GA & $\begin{array}{l}\text { Geologic Activity: Characterize current volcanic, tectonics, and sedimentary activity that modifies geologic units, } \\
\text { impact craters and ejecta. }\end{array}$ \\
\hline III.B.GW & Global Weathering: Determine the causes and spatial extent of global weathe ring regimes on Venus. \\
\hline
\end{tabular}

TECHNOLOGY DEVELOPMENT: Several challenging technologies should be developed to realize the full capabilities of the VeCaTEX mission concept:

- Aerobot Systems: Executing repeated trips to a region of the Venus atmosphere hotter than $100 \mathrm{C}$ presents aerobot design and materials challenges.

- Thermal control: Maintaining the NIR imaging systems at the optimal cool temperatures for acquiring high signal to noise imagers throughout the imaging traverse.

- Autonomy: Incorporating Sensing/Perception, State Estimation, Mission/Activity/Resource Planning and Scheduling, Fault Response, Diagnosis \& Prognosis [15] in the aerobot.

- Attitude Control: Aligning camera attitude with the path of the aerobot without the use of a celestial reference for optimal image acquisition.

PROGRAMMATIC RELATIONSHIPS: The VeCaTEx mission can benefit from data acquired by missions occurring during the next decade.

- Orbital Radar and Infrared Maps: A mission designed to acquire an improved global map of radar imaging and topography as well infrared surface signatures such as VERITAS or EnVision would allow the selection of a prioritized set of targets areas for observation.

- Improved Aerobot Trajectory Modeling: A variable-altitude (Type 2) aerobot operating in the mid cloud region as described in the Venus Flagship Mission concept study or the Thalassa and Venus Climate and Geophysical Mission Decadal Survey white papers would provide new information on atmospheric dynamics enabling improved path predictions for the aerobot. 
- Descent Probe or Dropsonde: A probe equipped with an infrared imaging system and capable of measuring cloud and haze properties in situ, would demonstrate below cloud nighttime imaging, determine the extent of sub-cloud haze layers and refine the design of the infrared cameras.

SUMMARY: VeCaTEx can transform knowledge of the origin and evolution of Venus as expressed in the youthful coronae and ancient tesserae. The mission concept directly addresses six priority investigations in the VEXAG GOI. It exploits advanced aerobot technologies that can be matured during the next decade according to VEXAG's Venus Strategic plan. VeCaTEx would naturally follow the next Venus surface-directed orbital mission and a mid-cloud aerobot mission with a launch early in the decade 2033-2042.

\section{REFERENCES}

[1] D. Dyar and N. Izenberg, "Venus Strategic Plan," VEXAG, 2020. [Online]. Available: https://www.Ipi.usra.edu/vexag/reports/Combined_VEXAG_Strategic_Documents_Current.pdf.

[2] K. Baines, G. Bellucci, J.-P. Bibring, B. Buratti and E. Bussoletti, "Detection of sub-micron radiation from the surface of Venus by Cassini VIMS," Icarus, vol. 148, pp. 307-311, 2000.

[3] R. W. Carlson, K. H. Baines, M. Girard, L. W. Kamp, P. Drossart, T. Encrenaz and F. W. Taylor, "Galileo/NIMS near-infrared thermal imagery of the surface of Venus," in Lunar and Planetary Science Conference 24 p 253, Houston, 1993.

[4] V. Meadows and D. Crisp, "Ground-based near-infrared observations of the Venus nightside: The thermal structure near the surface," J. Geophys. Res. , vol. 101, pp. 4595-4622, 1996.

[5] G. Hashimoto, M. Roos-Serote, S. Sugita, M. Gilmore, L. L. Kamp, R. Carlson and K. H. Baines, "Felsic highland crust on Venus suggested by Galileo Near Infrared Mapping Spectrometer (NIMS)," JGR-Planets, Vols. 114 E00B24, doi:10.1029/2008JE003134, 2009.

[6] S. Smrekar, E. Stofan, N. Mueller, A. Treiman, L. Elkins-Tanton, J. Helbert, G. Piccione and P. Drossart, "Recent Hotspot Vulcanism on Venus from VIRTIS data," Science, vol. 328, pp. 605-608, 2010.

[7] A. Gullcher, "Corona structures driven by plume-lithosphere interactions and evidence for ongoing plume activity on Venus," Nature Geoscience, 2020.

[8] M. Gilmore, N. Mueller and J. Helbert, "VIRTIS emissivity of Alpha Regio, Venus with implications for tessera composition," Icarus, no. 254, pp. 350-361, 2015.

[9] S. Smrekar, "VERITAS (VENUS EMISSIVITY, RADIO SCIENCE, INSAR, TOPOGRAPHY AND SPECTROSCOPY): A DISCOVERY MISSION," in Lunar and Planetary Science Conference, 2020.

[10] J. Helbert, "The Venus Emissivity Mapper - Obtaining Global Mineralogy of Venus from Orbit on the ESA EnVision and NASA VERITAS Missions to Venus," in Lunar and Planetary Science Conference, 2019.

[11] M. Gilmore, "Venus Flagship Mission Concept:," NASA Goddard Space Flight Center, 2020.

[12] J. Cutts, "Scientific Exploration of Venus with Aerial Platforms," National Academy of Sciences, 2020.

[13] R. G. Knollenberg and D. M. Hunten, "The Microphysics of the Clouds of Venus: Results of the Pioneer Venus Particle Size Spectrometer Experiment," JOURNAL OF GEOPHYSICAL RESEARCH, Vols. VOL. 85, , no. NO. Al3, pp. 8039-8058, 1980.

[14] C. Wilson, "Beyond Sulfuric Acid - What else is in he clouds of Venus," in Vensu Exploration Targets Workshop, 2014.

[15] A. Davis, "Imaging of infrared emission from the Venus surface from below the cloud base," JPL Report,2020.

[16] C. Bower, "Valriable Altitude Venus Platform," https//sbir.nasa.gov/SBIR/abstracts/20/sbir/phase1/SBIR-20-1-S3.055291.html, 2020.

[17] J. Hall and J. Izraelevitz, ". "Altitude-Controlled Light Gas Balloons for Venus Exploration," AIAA Aviation Forum, 2019.

[18] K. Baines, "Venus Climate and Geophysics Mission (VCGM: A Decadal Survey White Paper," JPL, 2020.

[19] P. Beauchamp and D. Dyar, "Venus Autonomoy Design Reference Mission," in SMDS Autonomy Conference, 2018. 